\title{
Role of Peripheral Sensory Neuron mu-Opioid Receptors in Nociceptive, Inflammatory, and Neuropathic Pain
}

\author{
Awinita Barpujari ${ }^{1}$, Neil Ford ${ }^{1}$, Shaoqiu He ${ }^{1}$, Qian Huang ${ }^{1}$, Claire Gaveriaux-Ruff ${ }^{2}$, \\ Xinzhong Dong ${ }^{1}$, Yun Guan ${ }^{3}$, and Srinivasa Raja ${ }^{1}$ \\ ${ }^{1}$ Johns Hopkins School of Medicine \\ ${ }^{2}$ Institut de Génétique et de Biologie Moléculaire et Cellulaire, Centre National de la \\ Recherche Scientifique/Institut National de la Santé et de la Recherche \\ Médicale/Université de Strasbourg \\ ${ }^{3}$ Johns Hopkins University School of Medicine
}

May 8, 2020

\begin{abstract}
Background and Purpose: The role of peripheral mu-opioid receptors (MOPs) in chronic pain conditions is not well understood. Here, we used a combination of mouse genetics, behavioral assays, and pharmacological interventions to investigate the contribution of MOPs on primary afferent neurons to nociceptive, inflammatory, and neuropathic pain as well as to opioid analgesia. Experimental Approach: We generated conditional knockout mice in which MOPs were selectively deleted in primary sensory neurons. Inflammatory and neuropathic pain states were induced in mutant and control wild-type mice and their behavioral responses to noxious stimuli compared. Gross motor function was also evaluated. Immunohistochemistry was used to assess MOP expression in the dorsal root ganglia, periaqueductal gray, and small intestine. Behavioral assays and whole-cell patch-clamp recordings were used to evaluate the inhibitory effects of mu-opioid agonists, DALDA and morphine, on pain behavior and neurophysiologic effects in DRG neurons, respectively. Key Results: Conditional MOP knockouts and control mice exhibited similar behavioral responses to acute nociceptive stimuli and developed similar inflammation-induced hypersensitivity. Nerve injury in animals lacking peripheral MOPs induced enhanced, bilateral mechanical allodynia. DALDA administered subcutaneously was unable to decrease the hypersensitivity induced by inflammation and nerve injury in MOP knockout animals, and morphine's antinociceptive effects were significantly attenuated in the absence of peripheral MOPs. Conclusion and Implication: MOPs in primary sensory neurons contribute to the modulation of neuropathic pain behavior and opioid analgesia. Our observations highlight the clinical potential of peripherally acting opioid agonists in the management of chronic inflammatory and neuropathic pain.
\end{abstract}

\section{Bullet Point Summary}

\section{What is already known}

- Opioid receptors in the central nervous system and periphery modulate inflammatory and neuropathic pain.

- Serious adverse effects of opioids result from their actions on MOPs in central nervous system.

\section{What this study adds}

- MOPs in peripheral sensory neurons play an important role in the modulation of nerve injury-induced neuropathic pain.

- The analgesic effects of systemic opioid agonists are attenuated in the absence of peripheral MOPs. 


\section{What is the clinical significance}

Opioid agonists targeting peripheral MOPs may have therapeutic benefits in nociceptive, inflammatory, and neuropathic pain.

Keywords: Opioid Receptors, Hyperalgesia, Neuropathic Pain, Inflammatory Pain, Dorsal Root Ganglion, Opioids

\section{Introduction}

Chronic inflammatory and neuropathic pains are complex clinical conditions associated with low quality of life and high economic burden (National Academies of Sciences, 2017). The management of pathological pain remains a clinical issue largely because the available treatments have limited efficacy and cause severe adverse effects. Though opioid analgesics are used to alleviate a myriad of pain conditions, their ability to penetrate the central nervous system (CNS) results in a variety of undesirable side effects, including respiratory depression, cognitive dysfunction, tolerance, and abuse (Dumas \& Pollack, 2008). In consideration of the ongoing opioid epidemic, safer analgesics that act outside the CNS are urgently needed.

A potential strategy to avoid CNS-induced drug effects may be to target opioid receptors in the peripheral nervous system. The use of peripherally acting opioid analgesics is supported by their inability to readily cross the blood-brain barrier and activate opioid receptors in the brain (Spahn et al., 2017; Xu et al., 2020). Mu-opioid receptors (MOPs), encoded by the Oprm1 gene, are the target of most opioid agonists, including morphine. They are expressed throughout ascending and descending pain pathways involved in pain transduction, modulation, and perception (Corder, Castro, Bruchas \& Scherrer, 2018). In the periphery, MOPs are localized in primary afferent (sensory) neurons of dorsal root ganglia (DRG). Their central and peripheral projections terminate in the spinal dorsal horn and somatic and visceral tissues, respectively. Primary sensory neurons play a critical role in initiating, transmitting, and modulating pain signals from the periphery. However, the contribution of peripheral MOPs to pain signaling and opioid pharmacology is unclear (Corder et al., 2017; Sun, Chen, Chen \& Pan, 2019; Weibel et al., 2013).

Experimental and clinical reports have shown that activation of peripheral opioid receptors produces analgesic and anti-inflammatory effects without causing centrally mediated side effects (Iwaszkiewicz, Schneider \& Hua, 2013; Machelska \& Celik, 2018). Studies of peripherally acting MOP agonists, such as loperamide and dermorphin [D-Arg2, Lys4] (1-4) amide (DALDA), provide pharmacological evidence for the importance of MOPs on primary afferents in the attenuation of tactile and thermal hypersensitivity in rodent models of neuropathic pain (Tiwari et al., 2016). Genetic disruption of MOPs in specific subpopulations of primary sensory neurons has allowed analysis of their contribution to distinct behavioral phenotypes. Strikingly, recent studies that have used the Cre/LoxP recombination system provide evidence that deletion of Oprm1 from primary sensory neurons prevents systemically administered opioids from inducing analgesia in acute and inflammatory pain conditions (Sun, Chen, Chen \& Pan, 2019).

In this study, we used transgenic mice in which MOPs had been deleted from $>90 \%$ of DRG neurons to assess their contribution to acute and chronic pain states. We used behavioral assays, immunohistochemistry, patch-clamp neurophysiology, in vivo pain models, and pharmacological interventions to evaluate the role of peripheral MOPs in opioid-induced analgesia.

\section{Methods}

\subsection{Animals}

Adult (4-6 months old) male and female wild-type (WT) C57BL/6J mice (20-45g; The Jackson Laboratory, Bar Harbor, ME) and Oprm1 conditional knockout mice (Oprm1 cKO) were housed under optimal laboratory conditions with a 12-hr light/dark cycle and free access to food and water. Mice were ear-marked at the time of weaning (21 days after birth), and tail biopsies were used for PCR genotyping. Mice were group- 
housed (one to five animals per cage) and maintained in a temperature- and humidity-controlled environment (18-26@C with 30-70\% relative humidity).

The Cre/LoxP recombination system was used to generate Oprm1 cKO mice in which MOPs were deleted specifically from primary sensory neurons. We crossed Oprm1 floxed mice, provided by Pr. Claire GaveriauxRuff (University of Strasbourg, Strasbourg, France), andPirt-Cre mice, provided by Dr. Xinzhong Dong (Johns Hopkins University, Baltimore MD, USA). Pirt is a modulator of several TRP channels and is expressed specifically in all primary sensory neurons in the DRG, but not in any CNS neurons. The Cre recombinase is under control of the Pirt promoter and expressed exclusively in $>95 \%$ of all DRG neurons (Kim et al., 2008). One WT allele of the Pirt gene must be present in order for the Cre recombinase to use the Pirt promoter (Pirt-Cre ${ }^{+/-}$). Animals homozygous for the floxed allele $\left(\mathrm{Oprm} 1^{\mathrm{f} / \mathrm{fl}}\right.$ ) and heterozygous for thePirt-Cre (Pirt-Cre ${ }^{+/-}$) transgene were selected for this study. Control WT animals were homozygous for both Oprm1 and Pirt (Pirt+/+ Oprm1 ${ }^{+/+}$).

Behavioral experiments were carried out with an equal number of age- and sex-matched adult WT and Oprm1 cKO mice. Animals were handled and acclimatized to laboratory conditions starting at least 1 week before being used in experimental procedures. All tests were carried out between 9:00 a.m. and 5:00 p.m. The experimental protocols were approved by the Animal Care and Use Committee of Johns Hopkins University and complied with the National Institutes of Health Guide for the Care and Use of Laboratory Animals to ensure minimal animal use and discomfort. Animals were randomly assigned into treatment groups, and investigators were blinded to treatment assignment and outcome assessment. The number of mice in each group is designated in the figure legends. The sample size in each group was determined based on our previous studies with similar experimental protocols (Tiwari et al., 2018; Tiwari et al., 2020). Animal studies are reported in compliance with the ARRIVE guidelines and with the recommendations made by the British Journal of Pharmacology(Kilkenny, Browne, Cuthill, Emerson \& Altman, 2010; McGrath, McLachlan \& Zeller, 2015).

\subsection{Inflammatory pain model}

We used complete Freund's adjuvant (CFA; Sigma-Aldrich, St Louis, MO) as a model of inflammatory pain. Baseline response to thermal stimulation was determined before CFA administration. Then, animals were anesthetized through isoflurane inhalation, and $20 \mu \mathrm{l}$ of $1 \mathrm{mg}[?] \mathrm{mL}^{-1}$ CFA was administered via subcutaneous (s.c.) injection into the plantar surface of the left hind paw with a 1-ml tuberculin syringe (BD, Franklin Lakes, NJ). CFA-induced paw edema and thermal hypersensitivity were assessed at 24 and $48 \mathrm{hr}$ postinjection.

\subsection{Edema measurement}

Paw thickness was used as a measure of inflammation-induced edema (Buritova, Honore \& Besson, 1995; Wei, Zou, Young, Dubner \& Ren, 1999). The dorsoventral thickness of each hind paw was measured with a digital caliper. The caliper was placed at the metatarsophalangeal border and touched, but did not squeeze, the hind paw. Contralateral paw thickness was used as a control measure.

\subsection{Neuropathic pain model}

The spared nerve injury (SNI) model of neuropathic pain was used. This partial denervation model involves ligation and excision of two of the three distal branches of the sciatic nerve. Traditional SNI involves ligating the common peroneal and tibial nerves and sparing the sural nerve (Decosterd \& Woolf, 2000). We used a variation in which the tibial nerve was spared $\left(\mathrm{SNI}_{t}\right)$ and the common peroneal and sural nerves were ligated and cut. Baseline responses to mechanical stimuli were determined before surgery. Then mice were deeply anesthetized with isoflurane 1.5-4.0\% (Abbott laboratories, North Chicago, IL), and toe pinch was used to verify that mice were under the surgical plane of anesthesia. Under aseptic conditions, the sciatic nerve was exposed at mid-thigh level through the biceps femoris muscle. The common peroneal and sural nerves were tightly ligated with 6.0 silk and axotomized distal to the ligation. Care was taken to avoid any contact with or stretching of the intact tibial nerve. Muscle and skin were closed in two layers with 4.0 silk 
and staples, respectively. In sham surgeries, the branches of the sciatic nerve were exposed but not ligated. The animals were returned to their cages after surgery and monitored during recovery. $\mathrm{SNI}_{\mathrm{t}}$ produces early $(<24 \mathrm{hr})$ and prolonged (>12 weeks) mechanical hypersensitivity and neuropathic pain predominantly in the area innervated by the tibial nerve (Tallarida, 2006). Behavioral experiments were initiated 2 days after surgery.

\subsection{Response to mechanical stimuli}

We evaluated mechanical hypersensitivity by measuring the hind paw withdrawal response to von Frey filament stimulation. Mice were placed individually in Plexiglas cages positioned on a wire-mesh stand. Mice were allowed to acclimate to the environment for approximately $30 \mathrm{~min}$ before testing. Low force $(0.07$ g) and high force $(0.45 \mathrm{~g})$ monofilaments (Stoelting Co., Wood Dale, IL) were applied to a fixed point on the midplantar surface of each hind paw (Guan, Liu, Tang, Raja, Anderson \& Dong, 2010). Each von Frey filament was applied 10 times successively at approximately l-2-s intervals. Vertical elevation of the paw and licking associated with the mechanical application were considered withdrawal responses. Paw withdrawal frequencies (PWF) were calculated for both hind paws.

\subsection{Response to thermal stimuli}

\subsubsection{Hot plate test}

Heat thresholds were measured in the hot plate test. Animals were placed in their home cages and allowed to acclimate for at least 30 min before testing. After habituation, an unrestrained animal was placed on the metal surface of the hot plate, which was maintained at a constant temperature of 55 . The response latency (i.e., the time before nocifensive behavior was exhibited) was recorded. Nocifensive behaviors included forepaw withdrawal or licking, hind paw withdrawal or licking, stamping, leaning posture, and jumping. If no behavioral response was elicited after $40 \mathrm{~s}$ (cutoff time), the animal was removed from the hot plate to prevent tissue damage. This procedure was repeated three times and the average across trials was used for data analysis.

\subsubsection{Hargreaves test}

Paw withdrawal latency to radiant heat was evaluated by the Hargreaves test (Hargreaves, Dubner, Brown, Flores \& Joris, 1988). Animals were placed individually in Plexiglas cages positioned on a heated glass floor $\left(30^{\circ} \mathrm{C}\right)$ and allowed to habituate for at least $30 \mathrm{~min}$ before testing. A radiant heat source was positioned underneath the animal and aimed at the plantar surface of each hind paw. The time interval before the animal withdrew its paw from the heat stimulus was measured by an automatic plantar stimulator meter (IITC model 390, Woodland Hills, CA) and recorded as the paw withdrawal latency (PWL). This test was repeated three times at 5-min intervals. If no behavioral response was elicited after $30 \mathrm{~s}$ (cutoff time), the stimulus was discontinued to avoid sensitization and prevent tissue damage. The average PWL across the three trials was used for data analysis.

\subsection{Motor function}

\subsubsection{Rotarod test}

Motor coordination, ataxia, and equilibrium were measured by using a rotarod apparatus (Ugo Basile, Varese, Italy). Mice were placed in the testing environment in their home cage and allowed to acclimate for at least $1 \mathrm{hr}$ to minimize effects of stress on behavior during testing. During a training session, mice from the same cage were placed in separate lanes on a rod rotating at $5 \mathrm{rpm}$ such that the animals were able to walk forward and keep their balance. After $60 \mathrm{~s}$, animals were returned to their home cage, and the apparatus was wiped with a cleaning solution. This procedure was repeated three times at 10-min intervals. If the animal fell off the rod before the 60 -s cutoff, the trial was repeated. However, no more than four trials were run per animal. Only the animals that were able to remain on the rod rotating at $5 \mathrm{rpm}$ for $60 \mathrm{~s}$ were used in the study. During the testing stage, animals from the same cage were placed in separate lanes on a rod that was initially rotating at $4 \mathrm{rpm}$. The apparatus was set to accelerate from 4 to $40 \mathrm{rpm}$ in $300 \mathrm{~s}$ (Deacon, 2013). The trial 
began when the acceleration started and ended when the animal fell from the rod. If the animal clung to the rod and completed a full passive rotation, the timer was stopped, and the passive rotation was noted. Care was taken not to disturb animals in adjacent lanes. This procedure was repeated for three trials. Latency to fall and speed at fall were recorded, and the averages of the three trials were used for data analysis.

\subsubsection{Open-field test}

Spontaneous exploration and locomotor activity were assessed in the open field test (Seibenhener \& Wooten, 2015). A Plexiglas arena (50 x $50 \mathrm{~cm}$ and $38 \mathrm{~cm}$ high) was equipped with a video tracking system (SMART 3, Panlab Harvard Apparatus, Spain) to monitor animal activity. The arena was wiped with $75 \%$ ethanol before and after each test to eliminate scents left by other mice. Mice were placed individually in the center of the apparatus at the beginning of each test and allowed to explore freely for $10 \mathrm{~min}$. Total distance traveled, mean travel speed, and number of center zone entries were determined by the software.

\subsection{Immunohistochemistry}

To assess the distribution of MOPs in the DRG and spinal cord, we performed immunofluorescence imaging. Animals, aged 6-8 weeks, were deeply anesthetized through isoflurane inhalation under saturated vapor pressure at room temperature, and perfused intracardially with $0.1 \mathrm{M}$ phosphate-buffered saline (PBS; $\mathrm{pH}$ $\left.7.4,4^{\circ} \mathrm{C}\right)$ followed by fixative $\left(4 \%\right.$ formaldehyde and $0.2 \%[\mathrm{v} / \mathrm{v}]$ picric acid in PBS, $\left.4^{\circ} \mathrm{C}\right)$. Tissues were cryoprotected in $30 \%$ sucrose for at least $24 \mathrm{hr}$ before being serially cut into $15-\mu \mathrm{m}$ sections and placed onto slides. Sections of spinal cord and lumbar DRG were cut on a cryostat and immunostained with guinea pig anti-MOP antibody (Neuromics, Cat \#GP10106, RRID:AB_2737108, 1:400). For secondary antibodies, we used Alexa 488-conjugated goat antibody to mouse (Thermo Fisher Scientific, Cat \#A-10667, RRID: AB_2534057) and Cy3-conjugated goat antibody to guinea pig (Thermo Fisher Scientific, Cat \#A-11073, RRID:AB_2534117). All secondary antibodies were diluted 1:100 in blocking solution (PBS $+1 \%$ bovine serum albumin $[\mathrm{BSA}]+0.1 \%$ Triton X-100). Images were taken at 40x with an AXIO Examiner.Z1 confocal microscope (Carl Zeiss AG, Oberkochen, Germany). Tissues from both experimental groups were processed simultaneously during the immunohistochemical process.

\subsubsection{Cell counting}

The number of immunopositive DRG neurons was determined by counting neurons that contained a nucleus and showed strong signal intensity in the cytoplasm $>2.5$-fold above the noise level in the same sections, as described previously (Nakamura et al., 2008). The total number of immunopositive neurons was calculated from two to three segment/level-matched DRG sections from 2-3 mice of each genotype.

\subsubsection{Signal intensity of MOP immunoreactivity in the spinal cord}

For measurements of MOP immunoreactivity, the spinal cord was divided along the dorsoventral axis and the signal intensity of each section was measured using ImageJ (version $1.52 \mathrm{v}, \mathrm{NIH}$ ). Superficial and deep lamina sections of Oprm1 cKO and WT mice (3 per group) were quantified by using a global background threshold and compared by their total integrated density (area $\times$ mean gray value).

\subsection{Genomic DNA analysis}

To detect the presence of the Oprm $1^{f l / f l}$ andPirt-Cre ${ }^{+/}$genes, we performed PCR on genomic DNA obtained from tail samples. Oprm1 primer sequences were 5'-GTTACTGGAGAATCCAGGCCAAGCC-3' (forward) and 5'-CGCTTGGGAATATCTTGTACCTATGACCA-3' (reverse). Pirt-Cre primer sequences were 5'ATCCGTAACCTGGATAGTGAA-3' (Cre forward), 5'-CAACTTTGTGGTACCCGAAG-3' (Pirt forward), and 5'-TCCCTGGGACTCATGATGCT-3' (Pirt reverse).

\subsection{Western blot analysis}

We used Western blot analysis to quantify MOP protein expression in lumbar DRG and spinal cord, small intestine, and periaqueductal gray. Samples of each tissue were separated and homogenized for immunoblotting. The tissues were lysed in ice-cold radioimmunoprecipitation assay buffer (50 mM Tris [pH 7.5], $150 \mathrm{mM}$ 
$\mathrm{NaCl}, 10 \%$ glycerol, $0.1 \%$ Triton X-100, $0.5 \mathrm{mg}[?] \mathrm{ml}^{-1} \mathrm{BSA}$ ). After samples were centrifuged at $13,000 \mathrm{~g}$ (or $12,000 \mathrm{rpm}$ ) for $15 \mathrm{~min}$, the protein concentration was determined by using a detergent-compatible protein assay (Pierce BCA Protein Assay Kit) with a BSA standard (Thermo Fisher Scientific, Waltham, MA). Samples were separated on a $7.5 \%(\mathrm{w} / \mathrm{v})$ sodium dodecyl sulfate-polyacrylamide gel by electrophoresis and transferred onto a nitrocellulose membrane (Amersham, Pittsburgh, PA) with a Trans-Blot Transfer Cell system (Bio-Rad, Hercules, CA). Membranes were incubated with the indicated primary antibody overnight at 4, and immunoreactivity was detected by enhanced chemiluminescence (ECL, Amersham). Antibodies against MOP (1:2000, AB5511, EMP Millipore) and GAPDH (1:50,000, ABS16, EMD Millipore) were used. Western blots were imaged with the ImageQuant LAS 4000 (GE Healthcare Life Sciences) and analyzed with ImageJ 1.46a software. GAPDH staining was used as an internal control for protein loading.

\subsection{Preparation of DRG neuronal cultures}

Primary cultures of DRG sensory neurons were obtained from naive, 3-4-week-old, male C57Bl/6 and Oprm1 cKO mice weighing $12-15 \mathrm{~g}$. Once the mice were anesthetized, the DRGs were carefully removed and immediately placed into fresh, ice-cold DH10 media consisting of DMEM/F-12 supplemented with 10\% fetal bovine serum (no DMSO) and $1 \%$ penicillin/streptomycin ( $\sim 1.5 \mathrm{ml}$ per animal), washed with Hanks' balanced salt solution (HBSS) 1-2x, and then treated with an enzyme solution containing $5 \mathrm{mg}[?] \mathrm{ml}^{-1}$ dispase and 1 $\mathrm{mg}[?] \mathrm{ml}^{-1}$ collagenase type I in HBSS without $\mathrm{Ca}^{2+}$ and $\mathrm{Mg}^{2+}$ (Gibco). Cells were shaken slowly for $>45$ $\mathrm{min}$ at $37 \mathrm{degC}$. After trituration (10-15x) and centrifugation, the cells were resuspended in DH10 media containing nerve growth factor $\left(50 \mathrm{U}[?] \mathrm{ml}^{-1}\right.$; Upstate Biotechnology) and plated on freshly made poly-Dlysine- (100 $\mu \mathrm{g}[?] \mathrm{ml}^{-1}$; Biomedical Technologies) and laminin- $\left(100 \mu \mathrm{g}[?] \mathrm{ml}^{-1}\right)$ coated coverslips. Cultures were then incubated at $37^{\circ} \mathrm{C}$ in $5 \% \mathrm{CO}_{2}$ until being used in subsequent electrophysiological studies.

\subsection{Whole-cell patch-clamp electrophysiology of cultured DRG neurons}

Whole-cell patch-clamp techniques were utilized to examine the electrophysiological and pharmacological properties of high-voltage-activated (HVA) calcium currents in DRG neurons from adult naïve mice. After a 48-72-hr incubation period, cultured DRG neuron preparations were placed into a submersion-type recording chamber (RC-22; Warner Instruments, Hamden CT, USA), secured to an inverted microscope, and visualized with a bright-field imaging system (Eclipse TE2000-U; Nikon). Patch-clamp electrodes were constructed from single-filament borosilicate glass $(1.5 \mathrm{~mm}$ outer diameter and $0.84 \mathrm{~mm}$ inner diameter; World Precision Instruments) with a microelectrode puller (P-1000; Sutter Instruments, Novato, CA). Electrode tip impedance ranged from 2 to $4 \mathrm{M} \Omega$ and formed seal resistances $>1 \mathrm{G} \Omega$ when filled with an internal recording solution composed of (in mM) 140 tetraethylammonium chloride, 10 EGTA, $1 \mathrm{MgCl}_{2}, 10$ HEPES, 0.5 GTP, and 3 ATP $(\mathrm{pH}=7.4$ by $1 \mathrm{M} \mathrm{N}$-methyl-D-glucamine [NMDG]; $300 \mathrm{mOsm}$, adjusted with sucrose, measured by a Wescor Vapro 5600, ELITech Group). Cultured neuron preparations were maintained under constant gravity-driven perfusion of an oxygenated external solution consisting of (in mM) 130 NMDG chloride, 5 $\mathrm{BaCl}_{2}, 1 \mathrm{MgCl}_{2}, 10 \mathrm{HEPES}$, and 10 glucose ( $\mathrm{pH}=7.4$ by $\mathrm{HCl} ; \sim \sim 210-315$ mOsm adjusted with sucrose), delivered at a rate of $1-2 \mathrm{ml}[?] \mathrm{min}^{-1}$ at room temperature. Tetraethylammonium chloride and NMDG chloride were added to each solution to block voltage-dependent $\mathrm{K}^{+}$conductance and $\mathrm{Na}^{+}$conductance, respectively. $\mathrm{Ba}^{2+}$ was added to the external solution to function primarily as a preferential charge carrier through the HVA channels, but also as a background $\mathrm{K}^{+}$conductance blocker. The junction potential between the internal and external solutions was not corrected for.

For patch-clamp recordings of HVA Ca ${ }^{2+}\left(\mathrm{HVA}_{\mathrm{Ca}}\right)$ currents in small diameter $(<20 \mu \mathrm{m}$ diameter $)$ DRG neurons, series resistance, if necessary was compensated and maintained at $<20 \mathrm{M} \Omega$ approximately $1-2$ min after the whole-cell configuration was established. The voltage protocol used to evoke HVA-I $\mathrm{I}_{\mathrm{Ca}}$ was modified from that of prior publications (Chen \& Ikeda, 2004; Li et al., 2017). Briefly, neurons were held at -80 mV, and a $40 \mathrm{mV}$ square wave voltage pulse was applied via the patch electrode (evoked to $-40 \mathrm{mV}$ ) for $20 \mathrm{~ms}$ to activate low-voltage-activated (LVA) $\mathrm{Ca}^{2+}$ channels. The holding voltage was then set at $-60 \mathrm{mV}$ for $20 \mathrm{~ms}$ followed by a $50 \mathrm{mV}$ voltage application delivered via the electrode (evoked to $-10 \mathrm{mV}$ ) for $20 \mathrm{~ms}$ to evoke HVA $\mathrm{Ca}^{2+}$ channels. After we recorded baseline HVA-I $\mathrm{Ia}_{\mathrm{Ca}}$ for $1 \mathrm{~min}$ to assess the stability of each evoked current, we applied either DALDA or morphine $(1 \mu \mathrm{M})$ to the neurons using a six-channel perfusion valve 
control system (VC-6; Warner Instruments) for a period of 2 min (time of full bath exchange) followed by a 5-min washout with the external solution. This HVA stimulation protocol was run every $10 \mathrm{~s}$ for a total of 8 min. Acquired recordings of $\mathrm{HVA}-\mathrm{I}_{\mathrm{Ca}}$ were filtered at $4 \mathrm{kHz}$ with a $-3 \mathrm{~dB}$, 4-pole, lowpass Bessel filter, sampled at a rate of $20 \mathrm{kHz}$, and stored on a personal computer (Dell) using pClamp 9.2 and a digitizer (Digidata 1322A, Molecular Devices). Offline, currents were digitally filtered by using a lowpass Gaussian filter with a $-3 \mathrm{~dB}$ cutoff set to $2 \mathrm{kHz}$ (Clampfit software; pClamp 9.2, Molecular Devices).

\subsection{Data and statistical analysis}

The data and statistical analysis comply with the recommendations of theBritish Journal of Pharmacology on experimental design and analysis in pharmacology (Curtis et al., 2018). Data are presented as the mean \pm standard errors of the mean (SEM). All data were subjected to a test of normality (ShapiroWilk or Kolmogorov-Smirnov), and either parametric or nonparametric analysis was conducted accordingly. Immunohistochemical and naïve behavioral data were compared across genotypes with Welch's t-test. The time course of drug and pain model effects was assessed by repeated-measures two-way ANOVA, followed by Bonferroni's multiple comparisons post hoc test. For immunoblot quantification, the amount of MOP protein was normalized to the mean of the WT control value, and the control value was set as 1 . All statistical analyses were performed with GraphPad Prism 8.0 software (Graph Pad Inc., San Diego, CA). All tests were two-tailed and the criterion of statistical significance was a probability less than $5 \%$.

\subsection{Materials}

\subsubsection{Drugs}

Morphine sulfate (Hospira, Inc., Lake Forest, IL) and the peripherally acting mu-opioid agonist DALDA (US Biological Life Sciences, Salem, MA) were diluted with normal saline and injected subcutaneously at 5 $\mathrm{mg}[?] \mathrm{kg}^{-1}$. Isoflurane (Sigma, St. Louis, MO) mixed in $2.5 \mathrm{l}[?] \mathrm{min}^{-1}$ oxygen was delivered through a nose cone to induce and maintain anesthesia and ensure the welfare of animals during surgical procedures. All drugs were weighed and prepared fresh prior to use. Drug doses were determined by their $\mathrm{ED}_{50}$ and are in line with our previous studies (Tiwari et al., 2018).

\subsubsection{Nomenclature of targets and ligands}

Key protein targets and ligands in this article are hyperlinked to corresponding entries in http://www.guidetopharmacology.org, the common portal for data from the IUPHAR/BPS Guide to PHARMACOLOGY (Harding et al., 2018), and are permanently archived in the Concise Guide to PHARMACOLOGY 2019/20 (Alexander et al., 2019).

Results 3.1 MOP expression is selectively deleted in DRG neurons and reduced in the spinal cord of Oprm1 cKO miceTo selectively delete MOP expression in primary sensory neurons, we crossed Oprm1 floxed mice and Pirt-Cre mice. LoxP sites were inserted upstream of Oprm1 exon 2 and downstream of exon 3 (Weibel et al., 2013). The Cre-mediated recombination deleted the floxed exons 2 and 3. Genotyping PCR was used to select animals that were heterozygous for the Pirt-Cretransgene (Pirt-Cre ${ }^{+/-}$) and homozygous for the nullOprm1 allele (Oprm1 $1^{\mathrm{f} / \mathrm{fl}}$; Figure 1$)$. Immunoblotting results indicated that MOP produced two protein bands (approximately 50 and $55 \mathrm{kDa}$ ) in all tissues tested from WT mice, potentially due to glycosylation (Huang, Chen \& Liu-Chen, 2015). In Oprm1 cKO mice, however, quantification analysis revealed a marked reduction in the level of MOP protein in the DRG (17.5\%), relative to that in WT mice. MOP protein expression levels in the spinal cord, small intestine, and periaqueductal gray tissues did not differ significantly between genotypes (Figure 2A, B). Immunofluorescence imaging of DRG sections showed that MOP antibody stained a significantly smaller subset of neurons in MOP cKO mice $(3.75+-$ 2.25 cells) relative to that in WT animals $(27.00+-2.75$ cells $)$. Immunofluorescence imaging of spinal cord sections revealed that MOP immunoreactivity was primarily distributed in the superficial laminae of the spinal dorsal horn in WT mice. In Oprm $1 \mathrm{cKO}$ mice, MOP immunoreactivity was significantly reduced to $58.91+-12.84 \%$ in the superficial dorsal horn and $75.88+-16.54 \%$ in deeper laminar neurons, relative to that in WT animals (Figure 2C-F) . MOP immunoreactivity was higher in deep laminae of cKO spinal cord 
than in superficial laminae. These data indicate that MOPs are removed exclusively from primary sensory neurons and their central terminals in the spinal cord of Oprm1 cKO mice.3.2 MOP agonists inhibit calcium currents in naive DRG neuronsPrior evidence suggests that MOP agonism diminishes neuronal excitability via G-protein-dependent inhibition of HVA calcium currents (Dolphin \& Scott, 1989; Rusin \& Moises, 1995; Tan, Groszer, Tan, Pandya, Liu \& Xie, 2003). To elucidate the involvement of MOP activation in DALDA application-dependent pain inhibition, we examined whole-cell patch-clamp electrophysiological recordings of HVA-I $\mathrm{I}_{\mathrm{Ca}}$ in adult WT mouse DRG neurons (Figure 3 ). Bath-application of $1 \mu \mathrm{M}$ DALDA (Figure 3A-C, top panels; $\mathrm{t}_{(146)}=11.84$, unpaired t-test) and $1 \mu \mathrm{M}$ morphine (Figure 3A-C, lower panels; $\mathrm{t}_{(106)}=16.75$, unpaired t-test) significantly inhibited HVA-I $\mathrm{I}_{\mathrm{Ca}}$ in naïve, small-diameter DRG neurons.3.3 Absence of MOPs in primary sensory neurons does not affect physiological response to noxious stimuli

WT and cKO mice showed no significant difference in behavioral response to acute thermal and mechanical stimuli. PWL to radiant heat was $14.90 \pm 1.43 \mathrm{~s}$ in WT mice and $14.72 \pm 0.78 \mathrm{~s}$ in Oprm1 cKO mice (Figure 4A ). In the hot plate test, response latency was $13.70 \pm 0.56 \mathrm{~s}$ in WT mice and $13.48 \pm 0.45$ $\mathrm{s}$ in the Oprm1 cKO group(Figure 4B). The von Frey assay for mechanical sensitivity (0.4 g filament) revealed a $35 \pm 2.24 \%$ PWF in WT mice and $34 \pm 2.21 \%$ PWF inOprm1 cKO mice (Figure 4C ). No sex differences in behavioral response to nociceptive stimuli were observed in either genotype (Supplemental Figure 1A-C) .

Additional studies confirmed that the absence of MOPs in primary sensory neurons does not affect gross motor function. Thus, the animals' behavioral response to nociceptive stimuli was not influenced by peripheral MOP-mediated motor deficits. Baseline motor coordination, ataxia, and balance, measured as the latency to fall from an accelerating rotarod, did not significantly differ between Oprm1 cKO and WT mice (Figure 4D) . Interestingly, significant differences were observed between groups in the open field test(Figure $\mathbf{4 E}, \mathbf{F}$ and Supplemental Figure 1E). WT mice traveled a total distance of $4135 \pm 199.40 \mathrm{~cm}$, whereas Oprm1 cKO mice traveled only $2660 \pm 175.00 \mathrm{~cm}$. WT mice spent approximately $7.52 \pm 1.02 \%$ of their time in the center of the open field apparatus, with an average of $67.92 \pm 10.79$ center zone entries, whereas Oprm1 cKO mice spent only $4.42 \pm 0.98 \%$ of their time in the center, with an average of $32.13 \pm 5.94$ entries. No significant differences were observed in the body weight of males or females between genotypes (not shown), and no sex-dependent differences were observed in motor activity (Supplemental Figure 1D-F) .

3.4 MOPs in primary sensory neurons are required for the antinociceptive effects of systemic DALDA and morphine

We next examined whether the absence of peripheral MOPs in primary sensory neurons affects the ability of opioids to block the response to acute nociceptive stimuli. In WT mice, the mean value for PWL to heat stimuli increased from $15.41 \pm 0.62 \mathrm{~s}$ at baseline to $18.05 \pm 0.69 \mathrm{~s}$, and the mean baseline PWF to mechanical stimulation decreased from $30 \pm 2.98 \%$ at baseline to $15 \pm 2.69 \%$ at 60 min after s.c. injection of $5 \mathrm{mg}[?] \mathrm{kg}^{-1}$ DALDA (Figure 5A) . In contrast, the same dose of DALDA produced no analgesic effect in the Oprm 1 cKO group. In WT mice, s.c. administration of $5 \mathrm{mg}[?] \mathrm{kg}^{-1}$ morphine significantly increased PWL from 14.41 +- $1.13 \mathrm{~s}$ to 19.79 +- $1.28 \mathrm{~s}$ and significantly reduced PWF from 33 +- $2.60 \%$ to 14 +$3.06 \%$ at 30 min post-injection (Figure 5B) . Morphine's analgesic effect in WT mice peaked at 30 min and persisted for 60 min post-administration. However, in the Oprm1 cKO group, s.c. injection of $5 \mathrm{mg}[?] \mathrm{kg}^{-1}$ morphine did not significantly affect the response to noxious mechanical and heat stimuli. These results suggest that peripheral MOPs in primary sensory neurons play an important role in the anti-nociceptive effect of systemically administered opioid agonists. No sex differences in the analgesic effects of DALDA or morphine were observed.

3.5 Inflammatory pain: MOPs in primary sensory neurons are essential for the analgesic effect of DALDA and contribute to analgesia from morphine

We sought to determine whether MOPs in primary sensory neurons mediate the analgesic effects of opioids on acute and persistent inflammatory pain. In WT mice, s.c. injection of $5 \mathrm{mg}[?] \mathrm{kg}^{-1}$ DALDA significantly 
reversed the CFA-induced inflammatory pain, as shown by an increase in PWL from 7.93 +- 0.69 s to 15.43 +$1.01 \mathrm{~s}, 60$ min after administration. Subcutaneous injection of the same DALDA dose produced no significant increase in PWL in the Oprm1 cKO group (Figure 6A). In WT mice, we found that s.c. injection of 5 $\mathrm{mg}[?] \mathrm{kg}^{-1}$ morphine induced an antinociceptive effect, in addition to an antihyperalgesic response, as PWL increased from a CFA-induced low of $6.68+-0.35 \mathrm{~s}$ to $18.34+-0.43 \mathrm{~s} 30 \mathrm{~min}$ after administration, compared to a pre-injury baseline PWL of $13.92+-0.73$ s(Figure 6B). Morphine's analgesic effect in WT mice peaked at $30 \mathrm{~min}$ and lasted for approximately $90 \mathrm{~min}$. In contrast, the same dose of morphine in Oprm $1 \mathrm{cKO}$ mice had a significantly smaller effect, increasing PWL from 5.81 +- $0.43 \mathrm{~s}$ to 9.33 +- $0.66 \mathrm{~s} 30$ min after administration, relative to a pre-injury baseline of $14.26+-0.74 \mathrm{~s}$. As in WT mice, the response to morphine peaked $30 \mathrm{~min}$ after drug administration. In the Oprm1 cKO group, the overall effect of morphine lasted for approximately $60 \mathrm{~min}$, and unlike in the WT cohort, did not fully reverse the CFA-induced reduction in PWL.

Additional studies confirmed that absence of MOPs in primary sensory neurons does not affect the development and severity of CFA-induced inflammation and thermal hyperalgesia. Response to thermal stimuli(Figure 6C) and paw edema measurements (Figure 6D)were evaluated before and at 24 and $72 \mathrm{hr}$ after CFA injection. No significant differences were observed between genotypes. Contralateral paw data showed that the effects of CFA were unilateral in both WT andOprm1 cKO mice. No sex differences were observed in the drug- and CFA-induced effects. These results verify that the drug effects were tested under comparable pain sensitivities in the two genotypes.

3.6 Neuropathic pain: Absence of MOPs in primary sensory neurons results in enhanced, bilateral, mechanical hypersensitivity after nerve injury

We investigated whether the absence of peripheral MOPs affects the development of $\mathrm{SNI}_{\mathrm{t}}$-induced mechanical allodynia. Before nerve injury, PWFs of left and right hind paws were similar and did not differ significantly between genotypes. At 2 days after nerve injury, ipsilateral PWF in response to high force mechanical stimuli had increased significantly in WT mice from $38+-3.27 \%$ to $74+-5.62 \%$ and in Oprm1 cKO mice from $32+-4.16 \%$ to 66 +- $5.62 \%$ (Figure 7). This nerve-injury induced increase in PWF was also observed in response to low force mechanical stimuli and persisted for up to 17 days post-injury. In the Oprm1 cKO group, withdrawal frequencies in the contralateral paw also increased significantly from preinjury baseline values. This effect peaked 5 days post-injury, with PWF in response to high force mechanical stimuli increasing significantly from $29+-2.77 \%$ at baseline to $56+-3.06 \%$. Notably, on day 5 , allodynia to high force mechanical stimuli contralateral to nerve injury inOprm1 $\mathrm{cKO}$ mice was as severe as that in the ipsilateral paw of WT mice. The WT group did not exhibit an injury-induced increase in contralateral PWF to mechanical stimuli. Tests with Oprm1 cKO sham-operated mice confirmed that the peripheral MOP-mediated bilateral mechanical allodynia was induced by nerve injury (Supplemental Figure 2A ).

Deletion of MOPs in primary sensory neurons did not impair gross motor function after nerve injury. Post$\mathrm{SNI}_{\mathrm{t}}$ rotarod activity did not differ between genotypes, and, when compared to their naive counterparts, neither genotype showed significant differences in the open field test after nerve injury (Supplemental Figure 2B, C) . No significant differences were observed in the body weight of nerve-injured animals (not shown), and no sex-dependent differences were noted in their behavioral and motor activity (not shown).

3.7 Neuropathic Pain: MOPs in primary sensory neurons contribute to the antihyperalgesic effect of systemic opioid agonists

We sought to determine whether peripheral MOPs in primary sensory neurons mediate the analgesic effect of opioids under neuropathic pain conditions. In WT mice, s.c. injection of $5 \mathrm{mg}[?] \mathrm{kg}^{-1}$ DALDA reversed the $\mathrm{SNI}_{\mathrm{t}}$-induced hyperalgesia, as evidenced by a significant decrease in PWF to high force mechanical stimuli from $62+-5.93 \%$ to $23+-5.17 \% 60$ min after administration. The same dose of DALDA produced no significant analgesic effect in the Oprm1 cKO group (Figure 8A). Previous investigations have provided evidence for a peripheral component of the antinociceptive effect of systemic morphine in rodents with peripheral mononeuropathy and spinal nerve ligation-induced neuropathy (Kayser, Lee \& Guilbaud, 1995; 
Pertovaara \& Wei, 2001). In our study, s.c. injection of $5 \mathrm{mg}[?] \mathrm{kg}^{-1}$ morphine decreased PWF from a $\mathrm{SNI}_{\mathrm{t}}$-induced peak of $61+-2.77 \%$ to $18+-2.91 \% 30$ min after administration (Figure 8B) . Morphine's analgesic effect in WT mice peaked at $30 \mathrm{~min}$ and lasted for approximately $90 \mathrm{~min}$. The same dose of morphine in Oprm $1 \mathrm{cKO}$ mice induced an attenuated analgesic effect. Thirty minutes after drug injection, the Oprm 1 cKO group exhibited a decrease in ipsilateral PWF from $61+-3.79 \%$ to $35+-5.43 \%$. Similar to the time course in WT mice, morphine's peak response in Oprm 1 cKO mice occurred after 30 min. However, the overall effect of morphine in Oprm $1 \mathrm{cKO}$ mice lasted for only approximately $60 \mathrm{~min}$. Notably, morphine did not induce an analgesic response in the contralateral hind paw of Oprm1 cKO mice.

\section{Discussion and Conclusions}

Targeting MOPs in the peripheral nervous system represents a novel approach to the clinical management of acute and chronic pain. Critical to this practice, however, is a thorough understanding of these receptors' effects on ongoing pain-induced behavior and opioid analgesia. The selective deletion of MOPs in the DRG of primary sensory neurons enabled us to evaluate the functional contribution of these receptors to acute nociceptive, inflammatory, and neuropathic pain. By examining behavioral responses to noxious stimuli and the effects of exogenous opioid agonists, we found that the absence of MOPs in primary sensory neurons (1) does not alter the behavioral response to acute nociceptive stimuli or the development of inflammationinduced thermal hyperalgesia, (2) exacerbates nerve injury-induced mechanical allodynia, (3) abolishes the analgesic effect of DALDA, and (4) attenuates the analgesic effect of morphine.

In naive Oprm $1 \mathrm{cKO}$ and $\mathrm{WT}$ mice, MOP expression in intestine and periaqueductal gray was similar, as was paw withdrawal response to mechanical and thermal stimuli. Prior research has shown that MOP immunoreactivity in the gastrointestinal tract of rodents is confined primarily to the myenteric plexus and that MOP myenteric neurons are most numerous in the small intestine, followed by the stomach and proximal colon (Sternini, Patierno, Selmer \& Kirchgessner, 2004). Intestinal MOP expression was unaltered in ourOprm1 cKO animals. Global expression of MOP in the spinal cord (ventral and dorsal regions) did not decrease; however, MOP immunoreactivity was significantly reduced in the superficial laminae. This finding supports

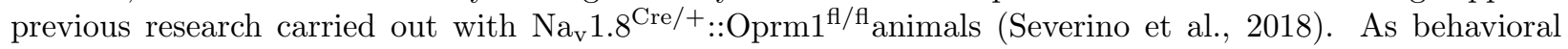
response and sensitivity to acute noxious stimuli were unaffected by the absence of peripheral MOPs, our findings suggest that endogenous tone at peripheral MOPs does not modulate response to acute noxious stimuli. These results are consistent with previous studies showing comparable baseline nociceptive sensitivity in $\mathrm{Na}_{\mathrm{v}} 1.8^{\mathrm{Cre} /+}:: \mathrm{Oprm}_{1}^{\mathrm{f} / \mathrm{fl}}$, TPRV1 ${ }^{\mathrm{Cre} /+}:: \mathrm{Oprm} 1^{\mathrm{f} / \mathrm{fl}}$, Advillin ${ }^{\mathrm{Cre} /+}:: O \operatorname{Oprm} 1^{\mathrm{f} / \mathrm{fl}}$, and control Oprm1 $1^{\mathrm{f} / \mathrm{fl}}$ animals (Corder et al., 2017; Martinez-Navarro et al., 2020; Severino et al., 2018; Sun, Chen, Chen \& Pan, 2019; Weibel et al., 2013)

By examining motor coordination, ataxia, and balance with the rotarod test, we showed that the absence of MOPs in primary sensory neurons does not affect gross motor function or ambulation under naive conditions. However, our findings from the open field exploration assay are worth noting. Both male and female MOP cKO mice exhibited significantly reduced spontaneous exploration and increased thigmotaxis (the tendency to remain close to walls), thereby mimicking behavior consistent with enhanced anxiety (Seibenhener \& Wooten, 2015). Previously, mice deficient in Preproenkephalin, anOprm1 pre-cursor gene, were found to display similar behavior, including increased anxiety and offensive aggressiveness (Konig et al., 1996).

Under naive conditions, the Oprm1 cKO mice, like Advillin ${ }^{\mathrm{Cre} /+}:$ Oprm $1^{\mathrm{f} / \mathrm{fl}}$ mice (Sun, Chen, Chen \& Pan, 2019), did not show opioid-induced analgesia during acute nociceptive stimuli. Although TRPV1- and Nav1.8-expressing DRG neurons have widespread distribution and overlap, neither TPRV1 ${ }^{\mathrm{Cre} /+}::$ Oprm1 ${ }^{\mathrm{f} / \mathrm{fl}}$

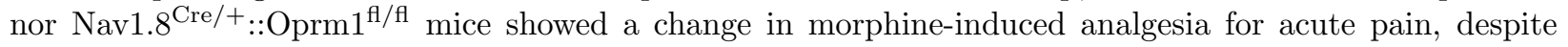
reduced MOP expression in the DRG. These results may be due in part to the different behavioral assays used to evaluate pain response (Corder et al., 2017; Weibel et al., 2013). Our observations suggest that an opioid-induced analgesic response to thermal and mechanical nociception may require MOP activation across multiple subpopulations of primary sensory neurons. As previously reported, MOPs present in TRPV1expressing neurons are primarily associated with opioid-induced hyperalgesia (i.e., opposing opioid analgesia), as the opioid analgesic effect is significantly enhanced when TRPV1-expressing neurons are removed (Chen 
\& Pan, 2006; Chen, Prunean, Pan, Welker \& Pan, 2007). Therefore, deletion of MOPs in TRPV1-expressing neurons may reveal that opioid-induced analgesia is a result of MOP activation in different subpopulations of DRG neurons.

In the CFA-induced pain model, Oprm1 cKO and WT animals developed comparable paw edema and thermal hyperalgesia, suggesting that MOPs in primary sensory neurons do not affect the inflammatory process or the subsequent development of inflammatory hyperalgesia. Of note, studies with $\mathrm{Na}_{\mathrm{v}} 1.8^{\mathrm{Cre} / \mathrm{fl}} \mathrm{MOP}$ animals showed that CFA-induced mechanical allodynia was comparable between groups until 1-week post-CFA. From 2 weeks post-CFA, MOP $\mathrm{Na}_{\mathrm{v}} 1.8$ cKOs exhibited enhanced sensitivity compared to their control counterparts (Severino et al., 2018).

In the periphery, endogenous mechanisms counteracting pain and inflammation arise from interaction between leukocyte-derived opioid peptides and opioid receptors on peripheral endings of primary afferent neurons, as well as anti-inflammatory cytokines (Celik et al., 2016; Labuz, Mousa, Schafer, Stein \& Machelska, 2007; Rittner et al., 2006; Shaqura, Zollner, Mousa, Stein \& Schafer, 2004; Stein \& Machelska, 2011; Stein, Schafer \& Machelska, 2003). Our observations suggest that the peripheral sensitization of nociceptors by inflammatory mediators such as prostaglandins, bradykinin, and other cytokines may be independent of the influence of neuronal MOP-mediated modulatory mechanisms.

We observed that Oprm1 cKO animals developed robust ipsilateral and contralateral mechanical allodynia after peripheral nerve injury, whereas WT mice exhibited only ipsilateral allodynia. This finding is consistent with our earlier studies, which showed similar bilateral mechanical allodynia after spinal nerve injury in global MOP knockout mice and in WT mice administered naloxone (Mansikka, Zhao, Sheth, Sora, Uhl \& Raja, 2004). Previous studies have also shown a bilateral increase in MOP binding in spinal dorsal horn of rats with unilateral chronic constriction injury-induced neuropathy (Stevens, Kajander, Bennett \& Seybold, 1991).

It is unclear what signaling mechanisms link the two sides of the body and mediate an effect on contralateral nonlesioned structures in the presence of peripheral neuropathy. Potential mechanisms of contralateral change have been differentiated based on CNS and peripheral nervous system signaling (Koltzenburg, Wall \& McMahon, 1999). In the periphery, it has been postulated that bilateral changes are mediated by circulating factors. This pathway would suggest that, after unilateral axotomy, breakdown products from the damaged nerve or denervated tissue may circulate in the bloodstream and induce changes in contralateral neuronal populations. Alternatively, contralateral effects may be governed by transmedian sprouting, possibly in the spinal cord. Thus, in midline structures and other tissues receiving bilateral inputs, unilateral nerve lesions might result in collateral sprouting of nearby and contralateral neurons. Such sprouting may also cause other changes in these contralateral neurons. Although additional studies are required to fully clarify the relationship between peripheral neuropathy and bilateral nociceptive signaling, our findings indicate that MOPs in primary sensory neurons play a critical role in the development of tonic bilateral inhibition of pain after nerve injury.

Previous investigations have shown that the analgesic effect of morphine on CFA-induced hypersensitivity is reduced in $\mathrm{Na}_{\mathrm{v}} 1.8^{\mathrm{Cre} /+}:: \mathrm{Oprm} 1^{\mathrm{H} / \mathrm{fl}}$ mice (Weibel et al., 2013). Similarly, we showed that the effects of morphine on inflammatory and neuropathic pain behaviors were attenuated in Oprm1 cKO mice, indicating that systemic opioids act in part via peripheral MOPs on sensory neurons. These data provide substantial evidence that MOPs in primary sensory neurons play a critical role in systemic opioid analgesia under conditions of inflammatory and neuropathic pain. Of note, one study (Sun, Chen, Chen \& Pan, 2019) reported that morphine was completely unable to induce analgesia in $O p r m 1 \mathrm{cKO}$ animals under inflammatory pain conditions. Though they observed a slight increase in paw withdrawal threshold, it was not statistically significant. The differences in our findings may be due to the different animal models used. Nonetheless, these results are in line with previously reported genetic and pharmacological data (Labuz, Mousa, Schafer, Stein \& Machelska, 2007; Weibel et al., 2013), which suggest that peripheral MOPs mediate mechanisms for certain doses of morphine. Moreover, our study is consistent with previous reports showing that peripheral nerve injury markedly reduces MOP expression in the DRG and represses the analgesic effect of systemically administered opioids via epigenetic mechanisms involving G9a and neuron-restrictive silence factor (Uchida, 
Sasaki, Ma \& Ueda, 2010; Xu et al., 2020).

Studies involving transgenic mouse models are commonly used to ascertain the role of distinct receptor mechanisms in pain signaling. We acknowledge that the evidence put forth by our Pirt-Cre ${ }^{+/-}:: O p r m 1^{\mathrm{f} / \mathrm{fl}}$ mice must be interpreted with caution, on account of potential confounding and/or compensatory factors. Deleting the Oprm1 gene in primary sensory neurons may lead to changes in the expression of other genes or nonspecific factors, thereby affecting the behavior of our transgenic animals. Moreover, the deletion of MOP expression in DRG neurons was dependent on the expression and efficiency of Cre. It is important to note that not all MOP-expressing DRG neurons express Cre and therefore were not under control of the Pirt promoter.

In summary, we assessed the functional role of MOPs in inflammatory and neuropathic pain using a new MOP-flox-Pirt-Cre mouse model that lacked these receptors in all primary sensory neurons. We found that these peripheral MOPs mediated all of the analgesia induced by the peripheral opioid DALDA in inflammatory and neuropathic pain states and attenuated the analgesia induced by morphine. These findings enhance our current understanding of the functional significance of peripheral MOPs and can aid in the development of potent new therapeutic agents that are devoid of the deleterious side effects associated with systemic opioids that cross the blood-brain barrier.

\section{Figure Legends}

Figure 1. Mouse genotypes. (A) Genetic makeup of mice used for this study: conditional knockout $(\mathrm{cKO})=$ Pirt-Cre $^{+/-}$Oprm1 $1^{\mathrm{f} / \mathrm{fl}}$ and wild-type $(\mathrm{WT})=\mathrm{Pirt}_{-\mathrm{Cre}}{ }^{+/+} \mathrm{Oprm}^{+/+}$.(B) Representative images of ethidium bromide-stained agarose gels show PCR genotyping of Pirt-Cre (top) and Oprm1 (bottom) from tail snip tissue samples. Higher band in Pirt-Crepanel indicates Pirt-Cre allele, and lower band indicates WT allele (i.e. Pirt gene without Cre $\left[\mathrm{Pirt}_{-} \mathrm{Cre}^{+/-}\right]$). Single band in $\mathrm{Oprm} 1$ panel indicates null gene (i.e. floxed allele $\left.\left[\mathrm{Oprm} 1^{\mathrm{f} / \mathrm{fl}}\right]\right)$.

Figure 2. Oprm1 conditional knockout (cKO) selectively deletes mu-opioid receptors (MOPs) in dorsal root ganglion (DRG) neurons. Representative western blot images (A) and quantification (B) of MOP protein levels in the DRG, spinal cord (SC), small intestine, and periaqueductal gray (PAG) (N=4-7 mice per group). The amount of MOP protein ( 50 and $55 \mathrm{kDa}$ ) was normalized to that of GAPDH (37 kDa) in the same sample, and the mean MOP level in wild-type (WT) mice was considered to be 1. Data are shown as means +- SEM. Protein levels were evaluated with Welch's t-test. Immunofluorescence-stained images and quantification of MOP expression in DRG $(\mathbf{C}, \mathbf{D})$ and spinal cord dorsal horn $(\mathbf{E}, \mathbf{F})$ in male Oprm1 ${ }^{\mathrm{f} / \mathrm{fl}}$ Pirt-Cre $^{+/-}$and WT mice ( $\mathrm{N}=2-3$ mice per group, 2-3 DRG sections per mouse). Arrow direction indicates dorsal side. Scale bar for DRG: $20 \mu \mathrm{m}$, and scale bar for spinal cord: $50 \mu \mathrm{m} .{ }^{*} p<0.05$.

Figure 3. Bath application of DALDA and morphine reduces the mean amplitude of HVA-I $\mathrm{I}_{\mathrm{Ca}}$ in cultured small-diameter DRG neurons. (A) Representative traces of HVA-I $\mathrm{I}_{\mathrm{Ca}}$ in response to bath application of 1 $\mu \mathrm{M}$ DALDA (Upper panel, $\mathrm{N}=4$; Black trace, pre-DALDA mean HVA-I $\mathrm{Ca}$ from 0-60 s; Gray trace, postDALDA mean HVA- $\mathrm{I}_{\mathrm{Ca}}$ from 120-145 s) and $1 \mu \mathrm{M}$ morphine (Lower panel, $\mathrm{N}=3$; Black trace, pre-morphine mean HVA-I $\mathrm{I}_{\mathrm{Ca}}$ from 0-60 s; Gray trace, post-morphine mean HVA-I $\mathrm{I}_{\mathrm{Ca}}$ from 120-145 s). Scale bars: 200 pA, $10 \mathrm{~ms}$.(B) Upper panel: A single bath application of $1 \mu \mathrm{M}$ DALDA produced a mean reduction in HVA- $\mathrm{I}_{\mathrm{Ca}}$ magnitude. Lower panel: A single bath application of $1 \mu \mathrm{M}$ morphine produced a mean reduction in HVA-I $\mathrm{Ia}_{\mathrm{a}}$ magnitude. Horizontal black bars indicate the duration of drug exposure. (C) Upper panel: Bath application of $1 \mu \mathrm{M}$ DALDA significantly reduced $\mathrm{HVA}-\mathrm{I}_{\mathrm{Ca}}\left(\mathrm{t}_{(146)}=11.84\right.$, unpaired t-test $)$. Lower panel: Bath application of $1 \mu \mathrm{M}$ morphine significantly reduced HVA- $\mathrm{I}_{\mathrm{Ca}}\left(\mathrm{t}_{(106)}=16.75\right.$, unpaired $\mathrm{t}$-test). ${ }^{*} p<0.05$.

Figure 4. Absence of mu-opioid receptors (MOPs) in primary sensory neurons does not affect behavioral response to acute pain.(A) Paw withdrawal latency in response to radiant heat stimulus. (B) Response latency to hot plate. (C) Paw withdrawal frequency in response to low force $(0.07 \mathrm{~g})$ and high force $(0.4$ g) von Frey monofilaments. (D) Latency to fall during rotarod test. (E) Total distance traveled and (F)number of entries into the center of the open field test apparatus by Oprm1 cKO and wild-type (WT) 
mice. (A-D) $\mathrm{N}=10 ;(\mathrm{E}, \mathrm{F}) \mathrm{N}=16$. Equal numbers of male and female mice were used. Data are shown as means \pm SEM and were compared by Welch's t-test. ${ }^{*} p<0.05$.

Figure 5. Systemic DALDA and morphine do not inhibit responses to acute nociceptive stimuli in MOP cKO mice. Wild-type and MOP cKO (Oprm1 $\left.{ }^{\mathrm{f} / \mathrm{fl}} \mathrm{Pirt}_{-\mathrm{Cre}}{ }^{+/-}\right)$mice were injected subcutaneously with 5 $\mathrm{mg}[?] \mathrm{kg}^{-1}$ DALDA or morphine. Time course of the effects of DALDA (A) and morphine(B) on the paw withdrawal latency in response to radiant heat stimuli and the paw withdrawal frequency in response to high force $(0.4 \mathrm{~g})$ von Frey filaments. $\mathrm{N}=10$ with 5 males and 5 females. Data are shown as means +- SEM. ${ }^{*} p<0.05$ compared to pre-drug (within the same genotype); \#p<0.05 compared to wild-type (same time point) by repeated measures two-way ANOVA with Bonferroni's post hoc test.

Figure 6. Absence of peripheral MOPs abolishes analgesic effect of systemic DALDA and attenuates analgesic effect of systemic morphine under persistent inflammatory pain conditions. (A , B ) Wild-type and MOP cKO (Oprm1 ${ }^{\mathrm{f} / \mathrm{f}}$ Pirt-Cre $\left.{ }^{+/-}\right)$mice were injected subcutaneously with $5 \mathrm{mg}[?] \mathrm{kg}^{-1}$ DALDA or morphine. Time course of the effects of DALDA (A) and morphine (B) on paw withdrawal latency in response to radiant heat stimuli. $\mathrm{N}=10$, with 5 males and 5 females. (C , D ) Wild-type and MOP cKO mice received a $20-\mu \mathrm{L}$ subcutaneous injection of $1 \mathrm{mg}[?] \mathrm{mL}^{-1} \mathrm{CFA}$ in one hind paw. Time course of the effects on thermal hyperalgesia(C) and paw edema (D). Data are shown as means \pm SEM. ${ }^{*} p<0.05$ compared to pre-drug or pre-CFA (within the same genotype); $\# p<0.05$ compared to wild-type (same time point) by repeated measures two-way ANOVA with Bonferroni's post hoc test.

Figure 7. Nerve injury induces bilateral mechanical allodynia in the absence of peripheral MOPs. Time course of the effects of $\mathrm{SNI}_{\mathrm{t}}$-induced neuropathic pain on ipsilateral and contralateral paw withdrawal frequency in response to (A) low force $(0.07 \mathrm{~g})$ and $(\mathbf{B})$ high force $(0.4 \mathrm{~g})$ von Frey monofilaments in Oprm1 cKO and wild-type mice. $\mathrm{N}=10$ with 5 males and 5 females. Data are shown as means \pm SEM. ${ }^{*} p<0.05$ (ipsilateral) and $+p<0.05$ (contralateral) compared to pre-surgery (within the same genotype);\# $p<0.05$ (ipsilateral) and $++p<0.05$ (contralateral) compared to wild-type (same time point) by repeated measures two-way ANOVA with Bonferroni's post hoc test.

Figure 8. Absence of peripheral MOPs abolishes analgesic effect produced by systemic DALDA and attenuates analgesic effect produced by systemic morphine in mice with neuropathic pain. Wild-type and MOP cKO $\left(\mathrm{Oprm}^{\mathrm{f} / \mathrm{fl}}\right.$ Pirt-Cre $\left.{ }^{+/-}\right)$mice were injected subcutaneously with $5 \mathrm{mg}[?] \mathrm{kg}^{-1}$ DALDA or morphine. Time course of the effects of DALDA (A) and morphine(B) on paw withdrawal frequency in response to low force $(0.07 \mathrm{~g})$ and high force $(0.4 \mathrm{~g})$ von Frey monofilaments. $\mathrm{N}=10$, with 5 males and 5 females. Data are shown as means + - SEM. ${ }^{*} p<0.05$ (ipsilateral) and $+p<0.05$ (contralateral) compared to pre-drug (within the same genotype); $\# p<0.05$ (ipsilateral) and $++p<0.05$ (contralateral) compared to WT (same time point) by repeated measures two-way ANOVA with Bonferroni's post hoc test.

\section{References}

Alexander SPH, Christopoulos A, Davenport AP, Kelly E, Mathie A, Peters JA, et al. (2019). THE CONCISE GUIDE TO PHARMACOLOGY 2019/20: G protein-coupled receptors. Br J Pharmacol 176 Suppl 1: S21-S141.

Buritova J, Honore P, \& Besson JM (1995). Indomethacin reduces both Krox-24 expression in the rat lumbar spinal cord and inflammatory signs following intraplantar carrageenan. Brain Res 674: 211-220.

Celik MO, Labuz D, Henning K, Busch-Dienstfertig M, Gaveriaux-Ruff C, Kieffer BL, et al.(2016). Leukocyte opioid receptors mediate analgesia via $\mathrm{Ca}(2+)$-regulated release of opioid peptides. Brain Behav Immun 57: 227-242.

Chen H, \& Ikeda SR (2004). Modulation of ion channels and synaptic transmission by a human sensory neuron-specific G-protein-coupled receptor, SNSR4/mrgX1, heterologously expressed in cultured rat neurons. J Neurosci 24: 5044-5053.

Chen SR, \& Pan HL (2006). Loss of TRPV1-expressing sensory neurons reduces spinal mu opioid receptors 
but paradoxically potentiates opioid analgesia. J Neurophysiol 95:3086-3096.

Chen SR, Prunean A, Pan HM, Welker KL, \& Pan HL (2007). Resistance to morphine analgesic tolerance in rats with deleted transient receptor potential vanilloid type 1-expressing sensory neurons. Neuroscience 145: $676-685$.

Corder G, Castro DC, Bruchas MR, \& Scherrer G (2018). Endogenous and Exogenous Opioids in Pain. Annu Rev Neurosci 41: 453-473.

Corder G, Tawfik VL, Wang D, Sypek EI, Low SA, Dickinson JR, et al. (2017). Loss of mu opioid receptor signaling in nociceptors, but not microglia, abrogates morphine tolerance without disrupting analgesia. Nat Med 23: 164-173.

Curtis MJ, Alexander S, Cirino G, Docherty JR, George CH, Giembycz MA, et al. (2018). Experimental design and analysis and their reporting II: updated and simplified guidance for authors and peer reviewers. Br J Pharmacol 175:987-993.

Deacon RM (2013). Measuring motor coordination in mice. J Vis Exp: e2609.

Decosterd I, \& Woolf CJ (2000). Spared nerve injury: an animal model of persistent peripheral neuropathic pain. Pain 87: 149-158.

Dolphin AC, \& Scott RH (1989). Modulation of Ca2+-channel currents in sensory neurons by pertussis toxin-sensitive G-proteins. Ann N Y Acad Sci 560: 387-390.

Dumas EO, \& Pollack GM (2008). Opioid tolerance development: a pharmacokinetic/pharmacodynamic perspective. Aaps j 10: 537-551.

Guan Y, Liu Q, Tang Z, Raja SN, Anderson DJ, \& Dong X (2010). Mas-related G-protein-coupled receptors inhibit pathological pain in mice. Proc Natl Acad Sci U S A 107: 15933-15938.

Harding SD, Sharman JL, Faccenda E, Southan C, Pawson AJ, Ireland S, et al. (2018). The IUPHAR/BPS Guide to PHARMACOLOGY in 2018: updates and expansion to encompass the new guide to IMMUNOPHARMACOLOGY. Nucleic Acids Res 46:D1091-d1106.

Hargreaves K, Dubner R, Brown F, Flores C, \& Joris J (1988). A new and sensitive method for measuring thermal nociception in cutaneous hyperalgesia. Pain 32: 77-88.

Huang P, Chen C, \& Liu-Chen LY (2015). Detection of mu opioid receptor (MOPR) and its glycosylation in rat and mouse brains by western blot with anti-muC, an affinity-purified polyclonal anti-MOPR antibody. Methods Mol Biol 1230: 141-154.

Iwaszkiewicz KS, Schneider JJ, \& Hua S (2013). Targeting peripheral opioid receptors to promote analgesic and anti-inflammatory actions. Front Pharmacol 4: 132.

Kayser V, Lee SH, \& Guilbaud G (1995). Evidence for a peripheral component in the enhanced antinociceptive effect of a low dose of systemic morphine in rats with peripheral mononeuropathy. Neuroscience 64: 537-545.

Kilkenny C, Browne W, Cuthill IC, Emerson M, \& Altman DG (2010). Animal research: reporting in vivo experiments: the ARRIVE guidelines. Br J Pharmacol 160:1577-1579.

Kim AY, Tang Z, Liu Q, Patel KN, Maag D, Geng Y, et al. (2008). Pirt, a phosphoinositide-binding protein, functions as a regulatory subunit of TRPV1. Cell 133:475-485.

Koltzenburg M, Wall PD, \& McMahon SB (1999). Does the right side know what the left is doing? Trends Neurosci 22: 122-127.

Konig M, Zimmer AM, Steiner H, Holmes PV, Crawley JN, Brownstein MJ, et al. (1996). Pain responses, anxiety and aggression in mice deficient in pre-proenkephalin. Nature 383: 535-538. 
Labuz D, Mousa SA, Schafer M, Stein C, \& Machelska H (2007). Relative contribution of peripheral versus central opioid receptors to antinociception. Brain Research 1160: 30-38.

Li Z, Tseng PY, Tiwari V, Xu Q, He SQ, Wang Y, et al. (2017). Targeting human Mas-related G proteincoupled receptor X1 to inhibit persistent pain. Proc Natl Acad Sci U S A 114: E1996-e2005.

Machelska H, \& Celik MO (2018). Advances in Achieving Opioid Analgesia Without Side Effects. Front Pharmacol 9: 1388.

Mansikka H, Zhao C, Sheth RN, Sora I, Uhl G, \& Raja SN (2004). Nerve injury induces a tonic bilateral mu-opioid receptor-mediated inhibitory effect on mechanical allodynia in mice. Anesthesiology 100: 912-921.

Martinez-Navarro M, Cabanero D, Wawrzczak-Bargiela A, Robe A, Gaveriaux-Ruff C, Kieffer BL, et al. (2020). Mu and delta opioid receptors play opposite nociceptive and behavioural roles on nerve-injured mice. Br J Pharmacol 177:1187-1205.

McGrath JC, McLachlan EM, \& Zeller R (2015). Transparency in Research involving Animals: The Basel Declaration and new principles for reporting research in BJP manuscripts. Br J Pharmacol 172: 2427-2432.

Nakamura S, Senzaki K, Yoshikawa M, Nishimura M, Inoue K, Ito Y, et al. (2008). Dynamic regulation of the expression of neurotrophin receptors by Runx3. Development 135: 1703-1711.

National Academies of Sciences E, and Medicine; Health and Medicine Division; Board on Health Sciences Policy; Committee on Pain (2017). Pain Management and the Opioid Epidemic: Balancing Societal and Individual Benefits and Risks of Prescription Opioid Use. In Pain Management and the Opioid Epidemic: Balancing Societal and Individual Benefits and Risks of Prescription Opioid Use. eds Phillips J.K., Ford M.A., \& Bonnie R.J. National Academies Press (US): Washington (DC).

Pertovaara A, \& Wei H (2001). Peripheral effects of morphine in neuropathic rats: role of sympathetic postganglionic nerve fibers. Eur J Pharmacol 429: 139-145.

Rittner HL, Mousa SA, Labuz D, Beschmann K, Schafer M, Stein C, et al. (2006). Selective local PMN recruitment by CXCL1 or CXCL2/3 injection does not cause inflammatory pain. J Leukoc Biol 79: 10221032 .

Rusin KI, \& Moises HC (1995). mu-Opioid receptor activation reduces multiple components of high-threshold calcium current in rat sensory neurons. J Neurosci 15: 4315-4327.

Seibenhener ML, \& Wooten MC (2015). Use of the Open Field Maze to measure locomotor and anxiety-like behavior in mice. J Vis Exp: e52434.

Severino A, Chen W, Hakimian JK, Kieffer BL, Gaveriaux-Ruff C, Walwyn W, et al. (2018). Mu-opioid receptors in nociceptive afferents produce a sustained suppression of hyperalgesia in chronic pain. Pain 159: $1607-1620$.

Shaqura MA, Zollner C, Mousa SA, Stein C, \& Schafer M (2004). Characterization of mu opioid receptor binding and $\mathrm{G}$ protein coupling in rat hypothalamus, spinal cord, and primary afferent neurons during inflammatory pain. J Pharmacol Exp Ther 308: 712-718.

Spahn V, Del Vecchio G, Labuz D, Rodriguez-Gaztelumendi A, Massaly N, Temp J, et al. (2017). A nontoxic pain killer designed by modeling of pathological receptor conformations. Science 355: 966-969.

Stein C, \& Machelska H (2011). Modulation of peripheral sensory neurons by the immune system: implications for pain therapy. Pharmacol Rev 63: 860-881.

Stein C, Schafer M, \& Machelska H (2003). Attacking pain at its source: new perspectives on opioids. Nat Med 9: 1003-1008.

Sternini C, Patierno S, Selmer IS, \& Kirchgessner A (2004). The opioid system in the gastrointestinal tract. Neurogastroenterol Motil 16 Suppl 2: 3-16. 
Stevens CW, Kajander KC, Bennett GJ, \& Seybold VS (1991). Bilateral and differential changes in spinal $\mathrm{mu}$, delta and kappa opioid binding in rats with a painful, unilateral neuropathy. Pain 46: 315-326.

Sun J, Chen SR, Chen H, \& Pan HL (2019). mu-Opioid receptors in primary sensory neurons are essential for opioid analgesic effect on acute and inflammatory pain and opioid-induced hyperalgesia. J Physiol 597: 1661-1675.

Tallarida RJ (2006). An overview of drug combination analysis with isobolograms. J Pharmacol Exp Ther 319: $1-7$.

Tan M, Groszer M, Tan AM, Pandya A, Liu X, \& Xie CW (2003). Phosphoinositide 3-kinase cascade facilitates mu-opioid desensitization in sensory neurons by altering G-protein-effector interactions. J Neurosci 23: 10292-10301.

Tiwari V, Anderson M, Yang F, Tiwari V, Zheng Q, He SQ, et al. (2018). Peripherally Acting mu-Opioid Receptor Agonists Attenuate Ongoing Pain-associated Behavior and Spontaneous Neuronal Activity after Nerve Injury in Rats. Anesthesiology 128: 1220-1236.

Tiwari V, He SQ, Huang Q, Liang L, Yang F, Chen Z, et al. (2020). Activation of micro-delta opioid receptor heteromers inhibits neuropathic pain behavior in rodents. Pain 161: 842-855.

Tiwari V, Yang F, He SQ, Shechter R, Zhang C, Shu B, et al. (2016). Activation of Peripheral mu-opioid Receptors by Dermorphin [D-Arg2, Lys4] (1-4) Amide Leads to Modality-preferred Inhibition of Neuropathic Pain. Anesthesiology 124: 706-720.

Uchida H, Sasaki K, Ma L, \& Ueda H (2010). Neuron-restrictive silencer factor causes epigenetic silencing of Kv4.3 gene after peripheral nerve injury. Neuroscience 166:1-4.

Wei F, Zou S, Young A, Dubner R, \& Ren K (1999). Effects of four herbal extracts on adjuvant-induced inflammation and hyperalgesia in rats. J Altern Complement Med 5: 429-436.

Weibel R, Reiss D, Karchewski L, Gardon O, Matifas A, Filliol D, et al. (2013). Mu opioid receptors on primary afferent nav1.8 neurons contribute to opiate-induced analgesia: insight from conditional knockout mice. PLoS One 8: e74706.

Xu B, Zhang M, Shi X, Zhang R, Chen D, Chen Y, et al. (2020). The multifunctional peptide DN-9 produced peripherally acting antinociception in inflammatory and neuropathic pain via mu- and kappaopioid receptors. Br J Pharmacol 177: 93-109.

\section{Hosted file}

1.docx available at https://authorea.com/users/319666/articles/449380-role-of-peripheralsensory-neuron-mu-opioid-receptors-in-nociceptive-inflammatory-and-neuropathic-pain

\section{Hosted file}

2.docx available at https://authorea.com/users/319666/articles/449380-role-of-peripheralsensory-neuron-mu-opioid-receptors-in-nociceptive-inflammatory-and-neuropathic-pain

\section{Hosted file}

3.docx available at https://authorea.com/users/319666/articles/449380-role-of-peripheralsensory-neuron-mu-opioid-receptors-in-nociceptive-inflammatory-and-neuropathic-pain

\section{Hosted file}

4.docx available at https://authorea.com/users/319666/articles/449380-role-of-peripheralsensory-neuron-mu-opioid-receptors-in-nociceptive-inflammatory-and-neuropathic-pain

\section{Hosted file}


5.docx available at https://authorea.com/users/319666/articles/449380-role-of-peripheralsensory-neuron-mu-opioid-receptors-in-nociceptive-inflammatory-and-neuropathic-pain

\section{Hosted file}

6.docx available at https://authorea.com/users/319666/articles/449380-role-of-peripheralsensory-neuron-mu-opioid-receptors-in-nociceptive-inflammatory-and-neuropathic-pain

\section{Hosted file}

7.docx available at https://authorea.com/users/319666/articles/449380-role-of-peripheralsensory-neuron-mu-opioid-receptors-in-nociceptive-inflammatory-and-neuropathic-pain

\section{Hosted file}

8.docx available at https://authorea.com/users/319666/articles/449380-role-of-peripheralsensory-neuron-mu-opioid-receptors-in-nociceptive-inflammatory-and-neuropathic-pain 